\title{
SMART SENSOR SYSTEM APPLICATION: An Integrated Compass
}

\author{
Ronald J. W. T. Tangelder, Guido Diemel, Hans G. Kerkhoff \\ MESA Research Institute /University of Twente \\ P.O. Box 2177500 AE Enschede, The Netherlands
}

\begin{abstract}
A fully integrable electronic compass has been designed based on the pulse position method, using micro-machined fluxgate magnetic sensors. The compass has been designed to have an accuracy of one degree. The analogue and digital circuitry in the system fit on a single Sea-of-Gates array of $200 k$ transistors. Together with the sensors it will be combined on a single MCM.
\end{abstract}

Keywords: mixed-signal design, micro systems, fluxgate sensors

\section{Introduction}

A number of magnetic sensor systems for navigational use have been described until now [Pet86, Spr93, Hol94, Mal95, Got95], but none of them have been shown to function as a complete compass system on a single chip or multi chip module. The main problem being the requirement of sensors and mixed analogue and digital circuitry on a single Silicon substrate.

With the introduction of micro-machined fluxgate sensing elements with electroplated permalloy cores [Kaw95] this problem has been solved partly. These sensors are particularly suitable for applications in the earth's magnetic field range as they combine high sensitivity with good integrability[Got95, Kaw95], and can be produced in a CMOS compatible process or can otherwise be used in a Multi Chip Module (MCM) implementation.
Their operating principle ensures robust and reliable operation of a portable compass system. Above mentioned properties are very appropriate for a fully integrable electronic compass system.

\section{Global system overview}

An overview of the total system is shown in Figure 1. The electronic compass functions by measuring the magnetic field in a horizontal plane in two perpendicular directions. The angle to the magnetic north is calculated by taking the arctangent of the division of the two measurants.

The system comprises of a analogue front-end which excites the sensors with a triangular waveform and converts the resulting sensor output to measurable digital signals. The digital back-end performs the angle calculation and LCD control.

The system uses a multiplexing technique by exciting one sensor at a time. This reduces both momental power consumption and chip area since only one oscillator is needed. The supply voltage is currently 5 Volts, but can be scaled down to 3.5V. Both digital and analogue parts are implemented on the fishbone Sea-of-Gates Array [Fre94] (see Figure 2). The fishbone SoG consists of 4 quarters, each with circa $50 \mathrm{k}$ pmos/nmos pairs. It is mainly intended for digital applications, but can very well be used for analogue designs, too (see [Haa95], [Don94]). Capacitors can be made by putting the second metal layer above the first one. Very large capacitors $(>400 \mathrm{pF})$ and resistors should be realised, however, on the substrate of the MCM.

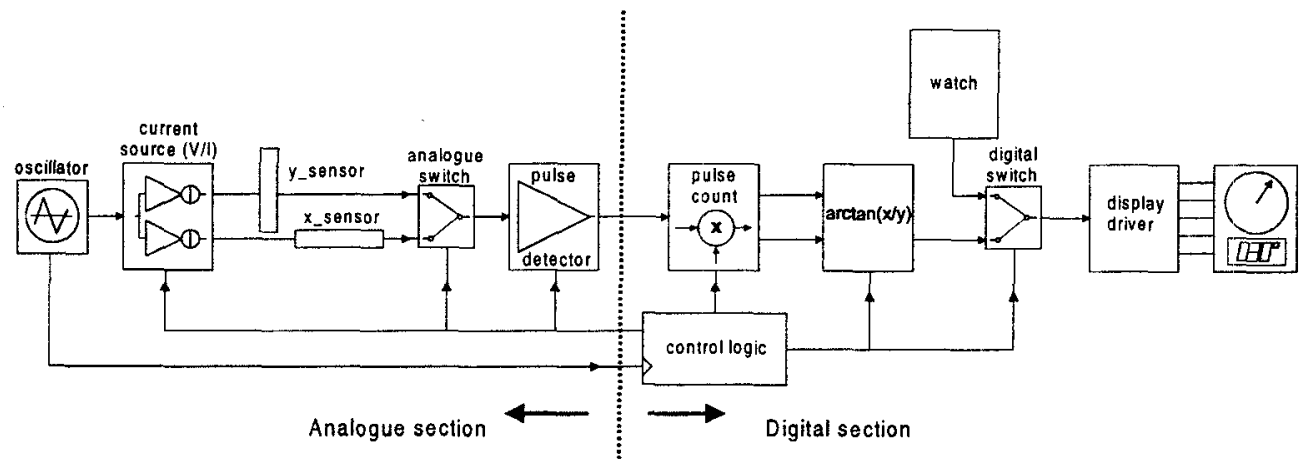

Figure 1: Overview of the electronic compass system 


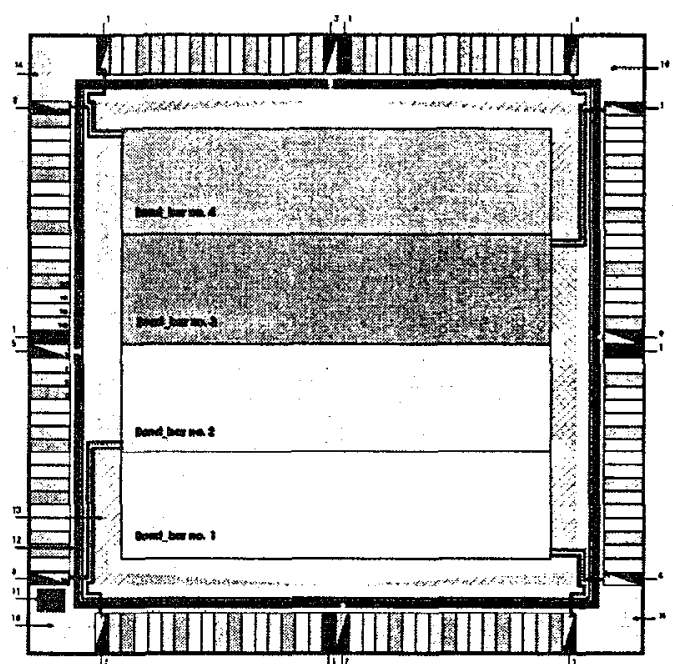

Figure 2: The fishbone Sea-of-Gates Array

The digital part of the integrated compass occupies 3 quarters fully and the analogue part 1 quarter for less than $15 \%$. Since each quarter has a separate power supply, we have used two different power supplies for both the digital and analogue parts.

The SoG and two micromachined sensors will be combined on a single $\mathrm{MCM}$, equipped with boundary scan test structures [Oli96].

\subsection{Sensors}

For fluxgate sensors several operating principles can be used. Most common is the so called second harmonic measurement [Rip92, Got95, Kaw95]. We, however, use the so called pulse position method [Rip92], which results in a very simple communication between the analogue and digital part [Hol94], as will be explained in subsection 3.2.

\subsubsection{Operating principle}

A fluxgate sensor is a form of transformer, which is deliberately driven into saturation periodically with a symmetrical excitation field (see Figure 3, solid line). If an external magnetic field $\mathrm{H}_{\mathrm{o}}$ is applied (the earth's magnetic field in this case), the sensor remains saturated longer in one direction and shorter in the other direction (see Figure 3, dashed line). The resulting change in induction voltage in the pickup coil is a measure for the external magnetic field.

If a triangular wave excitation field is applied, the induction voltage consists of pulses which shift in time due to the external field. An ideal sensor should reach saturation with an applied field with the same magnitude as the earth's magnetic field.

Measurements were carried out on an actual fluxgate sensor [Kaw95] and showed that it reached saturation at 15 times the magnitude of the earth's magnetic field $\left(\mathrm{H}_{\mathrm{K}}=10 \mathrm{Oe}\right)$. Also the internal resistance $(77 \Omega)$ of the sensor proved to be too high for low power applications.

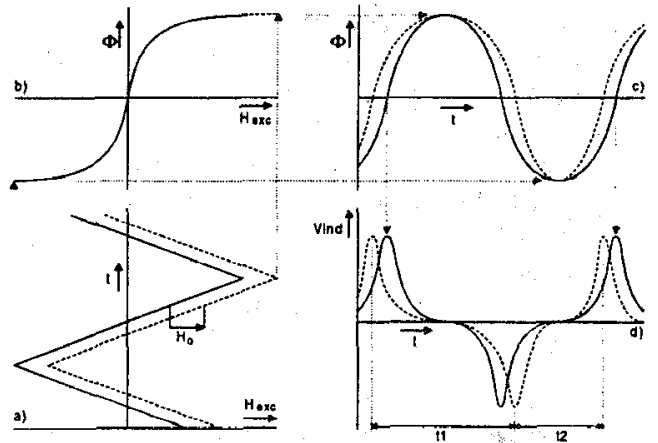

Figure 3: Pulse position operating principle of a fluxgate sensor, $H_{e x c} \equiv I_{e x c}$ and $V_{\text {ind }}=d \phi / d t$

An ELDO model was derived from these measurements. $H_{K}$ has been adapted to obtain a saturation level suitable for our application, which is still an obtainable goal for a new fluxgate sensor.

Hence, for the time being, a discrete miniaturised fluxgate sensor has been used. The resulting voltage over the pickup coil and the actual voltage over the excitation coil after applying a triangular excitation current of $12 \mathrm{~mA}$ peak to peak with a frequency of $8 \mathrm{kHz}$ have been measurements, and are displayed in Figure 4.
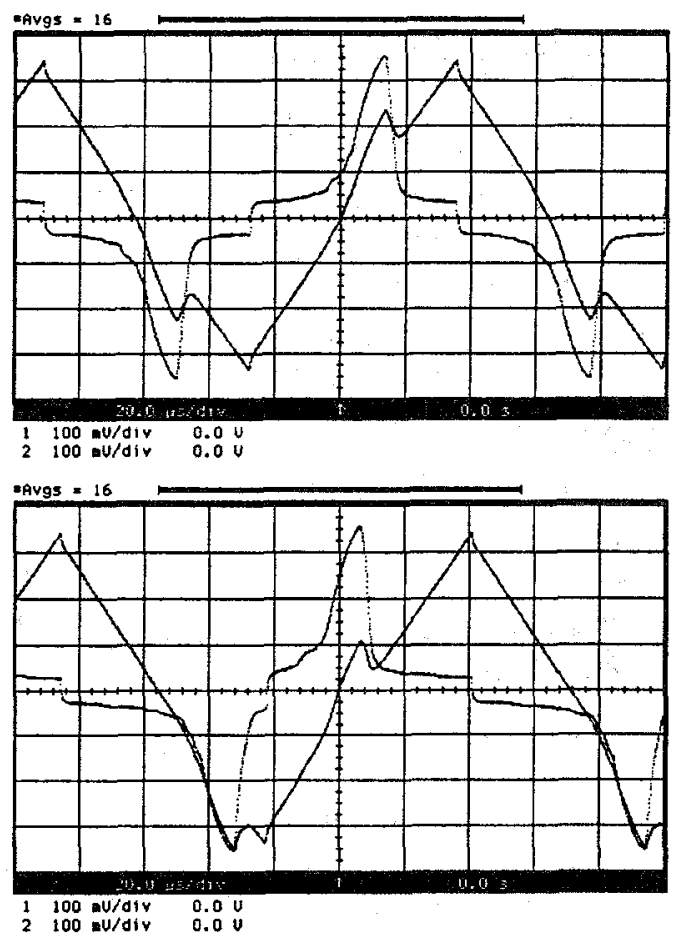

Figure 4: Real fluxgate sensor data, without and with a field applied.

The first measurement is without a field. In the second measurement a field is applied. The pulse shift is clearly visible. Notice also the change in impedance of the excitation coil, when saturation is reached. 


\subsubsection{Sensor structure}

The micromachined fluxgate sensor is based on a two-layer metalisation silicon process technology and ferromagnetic films.

A permalloy core is sandwiched between both metal layers as illustrated in Figure 5. This layout results in a transformer-like structure with a excitation coil and a pick-up coil.
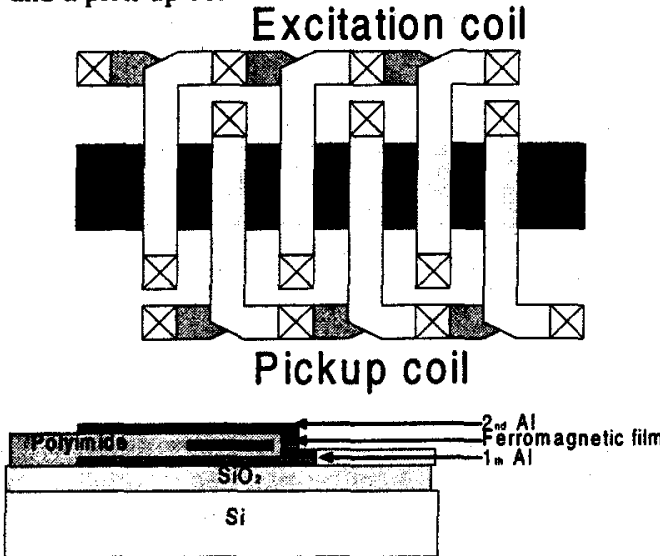

Figure 5 : Structure of the fluxgate sensing element

In Figure 6 a set of micromachined fluxgate sensing elements [Kaw95] is shown, each having a different number of windings.

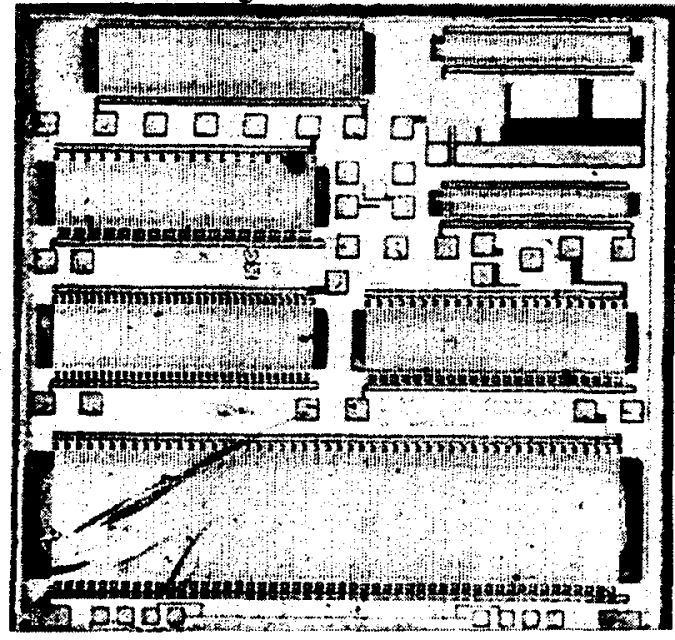

Figure 6: Fabricated micro-fluxgate sensing element [Kaw95]

\section{Analogue section}

The analogue section consists of two main parts: the excitation current source and the pulse position detector.

\subsection{The excitation current source}

The current source consists of a triangular waveform generator or oscillator and two VIconverters to drive the two sensors.

It has a triangular wave with an amplitude of $12 \mathrm{~mA}$ peak to peak, which is sufficiently high to drive the fluxgate sensors into saturation (see Figure 3a, b and c). The frequency of the waveform generated is $8 \mathrm{kHz}$..

The layout of the triangular waveform generator is given in Figure 7. It uses a capacitance of $10 \mathrm{pF}$ which is clearly visible in the upper half of the picture. An external resistor of $12.5 \mathrm{Meg} \Omega$, which is realised on the substrate of the MCM, should be connected to it.

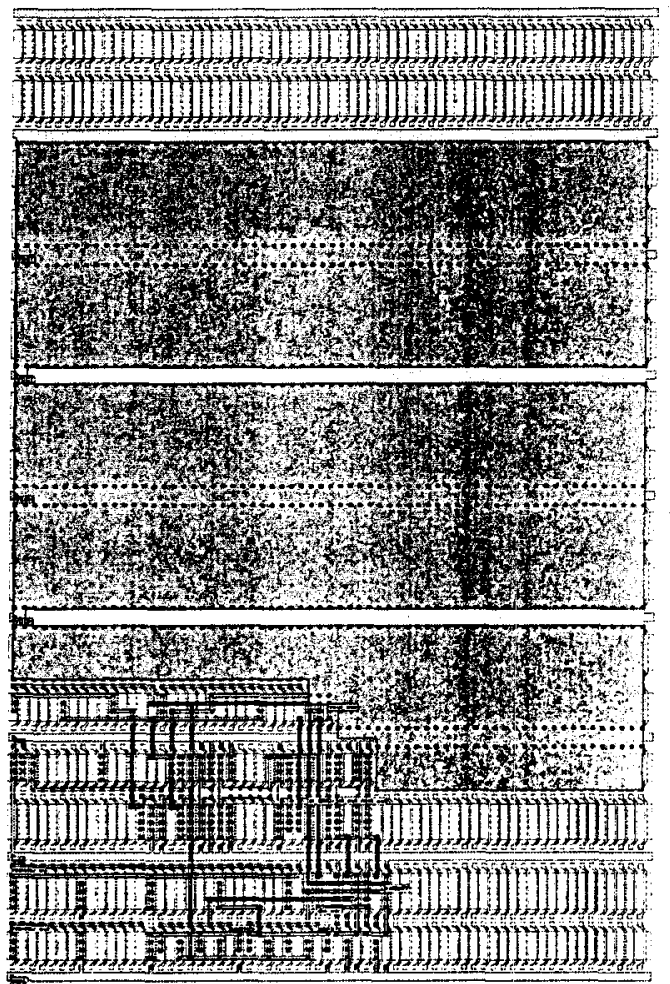

Figure 7 : Layout of the triangular waveform generator

Best sensitivity is obtained when the applied magnetic field is twice the saturation field. The linearity of the waveform is not very essential but the dc-offset is, and is therefore corrected by measuring the average of the excitation current. The sensors have a high series resistance, which requires the use of a balanced differential output. With the supply voltage at 5 Volt, sensors with a resistance as high as $800 \Omega$ can be driven. The resistive character of the sensors is used to linearise the excitation current sources.

\subsection{The pulse position detector}

The output of the sensor consists of voltage pulses which are detected in the second part (see Figure 3d and Figure 4).

Their position in time with respect to each other is measured by detecting both the falling edge of the positive pulse and the rising edge of the falling pulse. The pulse position detector processes a digital 1 after the falling edge of the positive pulse , which changes to a digital 0 after the rising edge of the negative pulse, and vice versa. 
This digital compatible signal which is sampled by a high speed digital up-down counter. The fraction of time in a period at which the output of the pulse detector is high is a direct indication of the field component measured. Since the analogue output consists only of one digital compatible signal, a complicated AD-converter is not necessary, which would have been the case for methods based on second harmonic measurements.

\section{Digital section}

The digital control logic has two main functions. It enables the analogue section and the digital high speed up-down counter only when they are needed, in order to diminish the power consumption further, and it controls the multiplexing of the two sensors.

The pulse count part contains a high-frequency $(4.194304 \mathrm{MHz})$ up-down counter, which transforms the output of the pulse detector into two integer values $x$ and $y$, each indicating the field component of the $x$ - and $y$-sensor.

The arctangent part gets an $x-$ and an $y$-value from the up-down counter and computes $\arctan (x / y)$, using a cordic-like algorithm (see Figure 8) [Spa76]. It used only 8 cycles to calculate the direction with an accuracy of one degree.

The calculation method is insensitive to local variations of the magnitude of the earth's magnetic field, which is necessary since the magnitude varies between $25 \mu \mathrm{T}$ in south America and $65 \mu \mathrm{T}$ near the south pole.

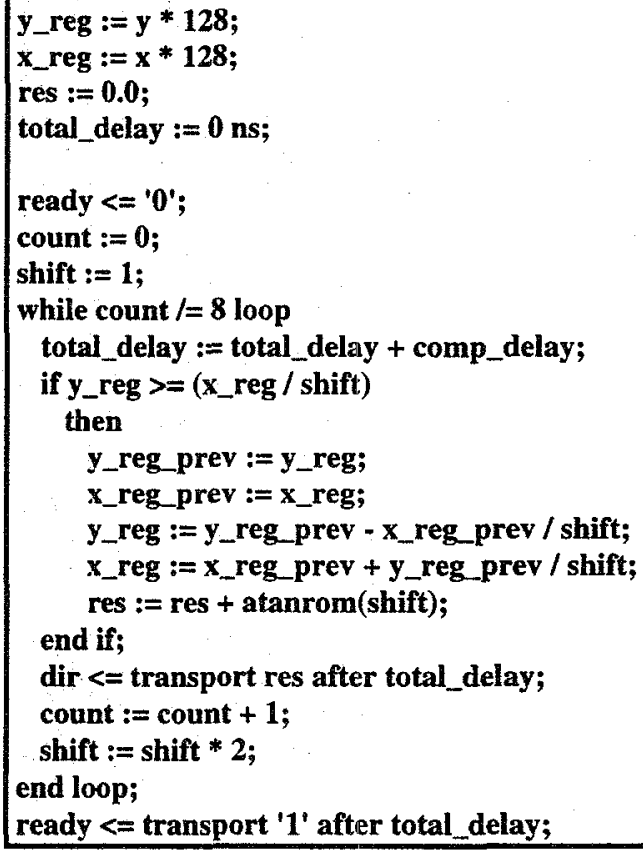

Figure 8: The main part of VHDL description of the arctan part.
The digital part contains also common watch options as added features. The display driver selects either the direction or the time to display. The pulse count part and the arctan part can be modified easily to compute the direction with an arbitrary precision. However, there will always be a bottle neck in the previous parts as the sensitivity of the fluxgate sensor and the analogue section are limited.

\section{Simulations and design flow}

Simulations were carried out with Compass Design Automation Tools and Anacad ELDO for the analogue and mixed-signal simulations. A model of the sensors based on realisable specifications was used.

The layout has been realised with the Ocean Design System [Gro93].

\section{Conclusions}

A miniaturised electronic compass system has been designed. The analogue and digital parts fit on a single Sea of Gates array. It combines sensing devices and analogue and digital circuitry in a single system by means of a Silicon based MCM. Simulations indicate that an accuracy within one degree is possible. The ideal sensor is not available yet but the system is designed to broad specifications so it can operate with fluxgate sensors which will be realised in near future.

\section{References}

[Pet86] T. J. Peters, "Automotive Navigation Using a Magnetic Flux-Gate Compass", IEEE Trans. Veh. Tech., VOL VT-35, No. 2, May 1986, pp 41-47

[Fre94] A. Frehe, "The division of the Sea of Gates Chip into 4 quarters", Internal report, Technical University Delft, the Netherlands

[Haa95] P.E. de Haan, "Analogue Circuit Design on Digital Sea-of-Gates Array", Ph.D. Thesis, University of Twente, September 1995, the Netherlands

[Don94] R. van Dongen and V.Rikking, "Advanced Analogue Circuit Design on a Digital Sea-ofGates Array", proc. ED\&TC 1994, pp. 70-74, Paris, France

[Oli96] J. Oliver, H.G. Kerkhoff, "Test Structures on MCM Active Substrate: Is it Worthwhile", proc. ED\&TC 1996, pp. 126-128, Paris, France

[Spr93] A. Sprotte, et al, "CMOS Magnetic-Field Sensor System”, proc. ESSCIRC 1993, 1993, pp 254-257

[Hol94] J. S. Hollema, et al, "Compass Watch, Report on the design of an Integrated Compass Watch", D1 project report, ICE, 1994, Twente University, The Netherlands

[Ma195] P. Malcovati, et al, "A Magnetic Sensor with Current-controlled Sensitivity and 
Resolution", Sensors and Actuators, A46-47, 1995, pp 284-288

[Got95] R. Gottfried-Gottfried, et al, "A Miniaturized Magnetic Field Sensor System Consisting of a Planar Fluxgate Sensor and a CMOS Readout Circuitry", Transducers '95, Sensors and Actuators, 289 - A 12, 1995, pp $229-232$

[Gro93] P. Groeneveld and P. Stravers, "Ocean: the Sea-of-Gates Design System Manual", 1994, Delft University of Technology, The Netherlands

[Kaw95]S. Kawahito, et al, "Micro-Fluxgate Magnetic Sensing Elements using Closely Coupled Excitation and Pickup coils", Transducers 1995, Sensors and Actuators, 290 - A12, 1995, pp 233 - 236

[Rip92]P. Ripka, "Review of fluxgate sensors", Sensors and Actuators A, 33, 1992, pp 129. 140

[Spa76] O. Spaniol, "Arithmetik in Rechenanlagen", Teubner Studienbücher Informatik, 1976 